\title{
Changes in Task Demands Alter the Pattern of zif268 Expression in the Dentate Gyrus
}

\author{
Elham Satvat, ${ }^{1 \star}$ Brandy Schmidt, ${ }^{2 \star}$ Melissa Argraves, ${ }^{2}$ Diano F. Marrone, ${ }^{1,3}$ and Etan J. Markus ${ }^{2}$ \\ ${ }^{1}$ Wilfrid Laurier University, Waterloo, Ontario N2L 3C5, Canada, ${ }^{2}$ University of Connecticut, Storrs, Connecticut 06269, and ${ }^{3} \mathrm{McKnight}$ Brain Institute, \\ University of Arizona, Tucson, Arizona 85719
}

Granule cells of the dentate gyrus (DG) are thought to disambiguate similar experiences - a process termed pattern separation. Using zif268 as a marker of cellular activity, DG function was assessed in rats performing two tasks: a place task (go east) and a response task (turn right). As these tasks occurred within the same physical space (a plus maze) without any physical cue to indicate the correct strategy in a given trial, this scenario critically involves disambiguation of task demands and presumably pattern separation. Performance of the two tasks induced zif268 expression in distinct populations of granule cells within the suprapyramidal but not the infrapyramidal blade of the DG. Repeated performance of the same task (i.e., two response-task trials or two place-task trials), however, elicited zif268 expression within a single subset of the granule cell population. This differential transcription pattern shows that the retrieval of different behavioral strategies or mnemonic demands recruit distinct ensembles of granule cells, possibly to prevent interference between memories of events occurring within the same physical space to permit the selection of appropriate responses.

\section{Introduction}

It is well established that place cells in the hippocampus respond with unique patterns of activity to different environments (O'Keefe and Nadel, 1978). Changes in these unique patterns (remapping) can be driven by changes in the environment, the nature of the behavioral tasks, or motivational states (Quirk et al., 1990; Bostock et al., 1991; Markus et al., 1995; Skaggs and McNaughton, 1998; Wood et al., 2000; Leutgeb et al., 2005; Wills et al., 2005; Kennedy and Shapiro, 2009). It is thought that formation of each distinct representation relies on pattern separation, a process by which differences between experiences are amplified to disambiguate similar events. Granule cells of the dentate gyrus (DG) may perform this pattern separation function (Marr, 1971; Treves and Rolls, 1992; Gilbert et al., 2001; Rolls and Kesner, 2006) due to their large number, sparse firing patterns (Jung and McNaughton, 1993; Leutgeb et al., 2007; Alme et al., 2010), and scant but powerful connections to CA3 (McNaughton and Morris, 1987; Amaral et al., 1990).

Remapping, however, has not been extensively studied in the DG, largely because of the technical challenge of recording cells in this region. These challenges may be overcome by using zif268 (AKA, egr 1), an immediate-early gene important for enduring plasticity and long-term memory (Jones et al., 2001), to label

Received Jan. 6, 2011; revised March 10, 2011; accepted April 7, 2011.

Author contributions: D.F.M. and E.J.M. designed research; E.S., B.S., and M.A. performed research; E.S., B.S., D.F.M., and E.J.M. analyzed data; E.S., B.S., D.F.M., and E.J.M. wrote the paper.

This work was supported by the Natural Sciences and Engineering Research Council of Canada (D.F.M.) and the Ontario Mental Health Foundation (D.F.M. and E.S.).

*E.S. and B.S. contributed equally to this work.

The authors declare no competing financial interests.

Correspondence should be addressed to Etan J. Markus, University of Connecticut, Department of Psychology, 406 Babbidge Road Unit 1020, Storrs, Connecticut 06269. E-mail: etan.markus@uconn.edu.

DOI:10.1523/JNEUROSCI.0094-11.2011

Copyright $\odot 2011$ the authors $\quad 0270-6474 / 11 / 317163-05 \$ 15.00 / 0$ place cells in the DG, since zif268 expression provides similar data to place cell recordings under comparable conditions. For instance, both methods demonstrate that granule cells are far more likely than expected by chance to be repeatedly active in the presence of multiple, highly distinct environments and that the pattern of granule cell activity is exquisitely sensitive to small changes in an animal's surroundings. For example, when exposed to two highly similar environments, the patterns of firing (Jung and McNaughton, 1993; Leutgeb et al., 2007) and zif268 expression (Marrone et al., 2010) in granule cells is far less correlated than in CA3 pyramidal cells. However, no study has explored how granule cells represent distinct experiences in a single, unaltered location in situations requiring disambiguation.

To address this question, rats were trained to navigate a plus maze twice daily with a $25 \mathrm{~min}$ break between sessions. Each session required a place (go east) or a response (turn right) strategy. Rats were trained to use the same strategy (place-place or response-response) or different strategies (place-response or response-place) on both sessions. After training using all starting locations, all trials on the final day started from the south arm, which results in an identical trajectory using either strategy. Using this paradigm, the effects of changing mnemonic demands on the pattern of zif268 expression can be assessed independently of changes in visual stimuli, trajectory, and motivational states. These data demonstrate that the DG disambiguates experiences occurring in a single spatial location.

\section{Materials and Methods \\ Subjects}

Twenty-two adult (11-14 months old) male Fischer-344 rats (Taconic) were used in this study. Rats were individually housed in Plexiglas cages $(46 \times 20 \times 23 \mathrm{~cm})$, maintained on a $12 \mathrm{~h}$ light/dark cycle, and food restricted to $85 \%$ of their ad libitum body weight. All procedures were 
a

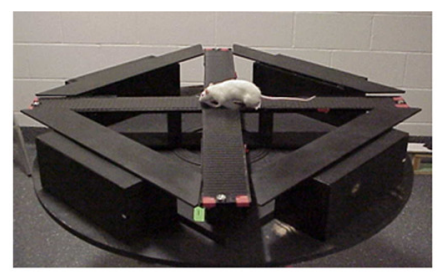

b

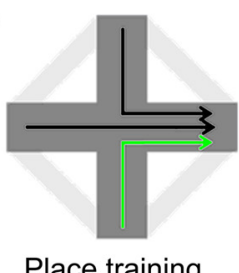

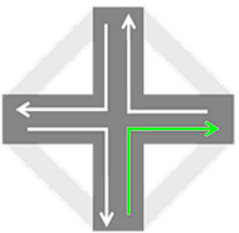

Response training
C

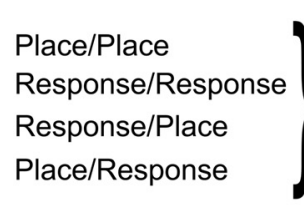

d

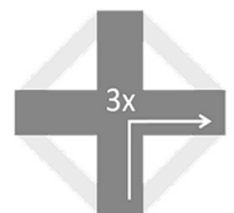

$25^{\prime}$

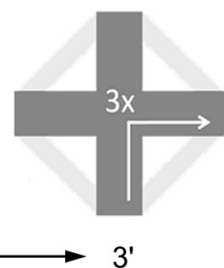

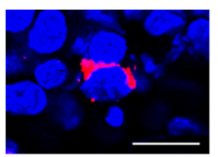
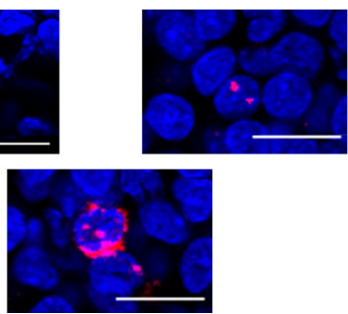

Figure 1. Schematic outline of the experiment. $\boldsymbol{a}, \boldsymbol{b}$, Rats were trained in a plus maze (a) to navigate to a target arm for food reward using either a place (i.e., go east) or a response (i.e., turn right) strategy $(\boldsymbol{b})$. Rats were trained to use one of four strategies: consistent spatial strategy (place-place), consistent response strategy (response-response), response strategy during the first session and a place strategy during the second (response-place), and place strategy during the first session and a response strategy during the second (place-response). Note that when the animal began from the south arm (green), both strategies resulted in an identical trajectory. $c$, During the final session, all groups were given two sessions of three trials on the maze beginning from the south arm, separated by a 25 min interval. $\boldsymbol{d}$, Following these trials, the animals' brains were harvested and the compartmental expression of zif268 (red) was measured to provide a histological record of activity in granule cells (blue) Scale bar, $20 \mu \mathrm{m}$. Cells active during the first epoch on the maze expressed zif268 within the cytoplasm (left); those active during the second epoch express zif268 within the nucleus (right). Granule cells active during both epochs express zif268 within both cellular compartments (bottom).

approved by the University of Connecticut Institutional Animal Care and Use Committee.

\section{Behavioral training}

Seventeen rats were trained as described previously (Schmidt et al., 2009). An additional five caged control rats were anesthetized with isoflurane and decapitated directly from their home cages.

Pretraining. Rats were trained to run a linear track $(120.7 \times 10.2 \mathrm{~cm})$ for chocolate sprinkle rewards daily. When rats completed 20 trials within $10 \mathrm{~min}$ for two consecutive days, training moved to a plus maze $(112.4 \times 10.8 \mathrm{~cm})$ with four perimeter runways (Fig. $1 a)$. Rats started each trial behind a removable transparent barrier, and the trial ended once the rat chose an arm. The rat was then guided through the perimeter runway to the next start arm. Training was on weekdays for a daily maximum of 32 correct trials or $20 \mathrm{~min}$, whichever occurred first.

Response or place training. Animals were randomly assigned a response or a place strategy task (Fig. 1b). Response task rats were trained to make a right hand turn relative to the start arm. Place task rats were required to go to the east arm, regardless of the start arm. Start arms were assigned pseudorandomly. If an error was made, the trial was repeated. Once each rat correctly completed $80 \%$ of trials over two consecutive days, reversal training began.

Reversal training. At this stage, training was divided into two daily sessions of 16 trials, with a 25 min intersession interval in which rats returned to their home cages. The animals in each group (i.e., place trained or motor response trained) were further divided; half of the rats continued to be trained on the same strategy, creating place-place $(\mathrm{P} / \mathrm{P}$; $n=4)$ and response-response ( $\mathrm{R} / \mathrm{R} ; n=4)$ groups. The remaining rats were trained during the second session to use the strategy not previously trained, creating place-response $(\mathrm{P} / \mathrm{R} ; n=4)$ and response-place $(\mathrm{R} / \mathrm{P}$; $n=5$ ) groups. The number of trials per session was reduced to five once each rat correctly completed $80 \%$ of trials for two consecutive days, and was further reduced to three once rats correctly completed $100 \%$ of trials for two consecutive days.

Final testing. On the final day of testing, the procedures were identical, with the exception that all trials started from the south arm. When begun at the south arm, all animal's trajectories were identical (i.e., rats completing a place trial were required to go to the east arm and rats completing the motor response trial were required to turn right, thus both strategies end in choosing the east arm). This ensured equivalent head orientations and sampling of the environment-important factors for zif268 expression (Marrone et al., 2010). If both sessions were performed perfectly, rats were immediately anesthetized with isoflurane and decap- itated. If an error was made, the rat was trained for at least two more training days using pseudorandomly chosen start arms, and another final day was then attempted.

\section{Histology}

After decapitation, brains were rapidly removed, flash-frozen in isopentane (Sigma-Aldrich), bathed in dry ice:ethanol, and stored at $-80^{\circ} \mathrm{C}$ before being shipped on dry ice to Wilfrid Laurier University. Using Tissue-Tek OCT compound (Fisher Scientific), the right hemispheres of eight rats were mounted together such that all conditions were represented. Coronal sections $(20 \mu \mathrm{m})$ were cut using a Leica CM3050 cryostat (Leica), thaw-mounted onto Superfrost-Plus slides (VWR), dried, and stored at $-80^{\circ} \mathrm{C}$.

\section{Fluorescence in situ hybridization}

Fluorescence in situ hybridization was performed as described previously (Guzowski et al., 1999). Briefly, zif268 riboprobes were synthesized using a transcription kit (Maxiscript; Ambion) and digoxigenin-labeled UTP (Roche Molecular Diagnostics), purified on RNA spin columns (Roche), and verified by electrophoresis. Once thawed, slides were fixed in $4 \%$ paraformaldehyde, treated with $0.5 \%$ acetic anhydride, incubated in methanol and acetone (1:1), equilibrated in saline-sodium citrate (SSC), and incubated with prehybridization buffer (Sigma-Aldrich) for $30 \mathrm{~min}$ at room temperature. Slides were then incubated in $100 \mathrm{ng}$ of riboprobe in a humid chamber overnight at $56^{\circ} \mathrm{C}$. This was followed by series of SSC washes and RNase A $(10 \mathrm{mg} / \mathrm{ml})$ at $37^{\circ} \mathrm{C}$ for $30 \mathrm{~min}$. Endogenous peroxidases were then quenched with $2 \% \mathrm{H}_{2} \mathrm{O}_{2}$. The tissue was blocked with TSA blocking buffer (PerkinElmer) containing normal sheep serum, and incubated with anti-digoxigenin-HRP antibody (Roche) for $2 \mathrm{~h}$ at room temperature. Slides were then washed in Tris-buffered saline with $0.05 \%$ Tween 20 and HRP-antibody conjugates were detected using a CY3 TSA kit (PerkinElmer). Tissue was then counterstained with DAPI (Sigma), coverslipped, and sealed with Vectashield (Vector Labs).

\section{Image acquisition and analysis}

Images were collected at $40 \times$ using an FV1000 confocal microscope (Olympus). Photomultiplier tube assignments, aperture size, and contrast values were kept constant for all sections on each slide. A series of images were acquired through each of the dorsal and ventral DG $(-2.80$ to -3.30 and -5.60 to -6.04 relative to bregma, respectively) (Paxinos and Watson, 2004). Within each region, $z$-stacks (optical sections, $\sim 1$ $\mu \mathrm{m}$; step size, $0.7 \mu \mathrm{m}$ ) were obtained from the suprapyramidal and infrapyramidal blades of the DG in two to three slides per animal (Fig. 2). 

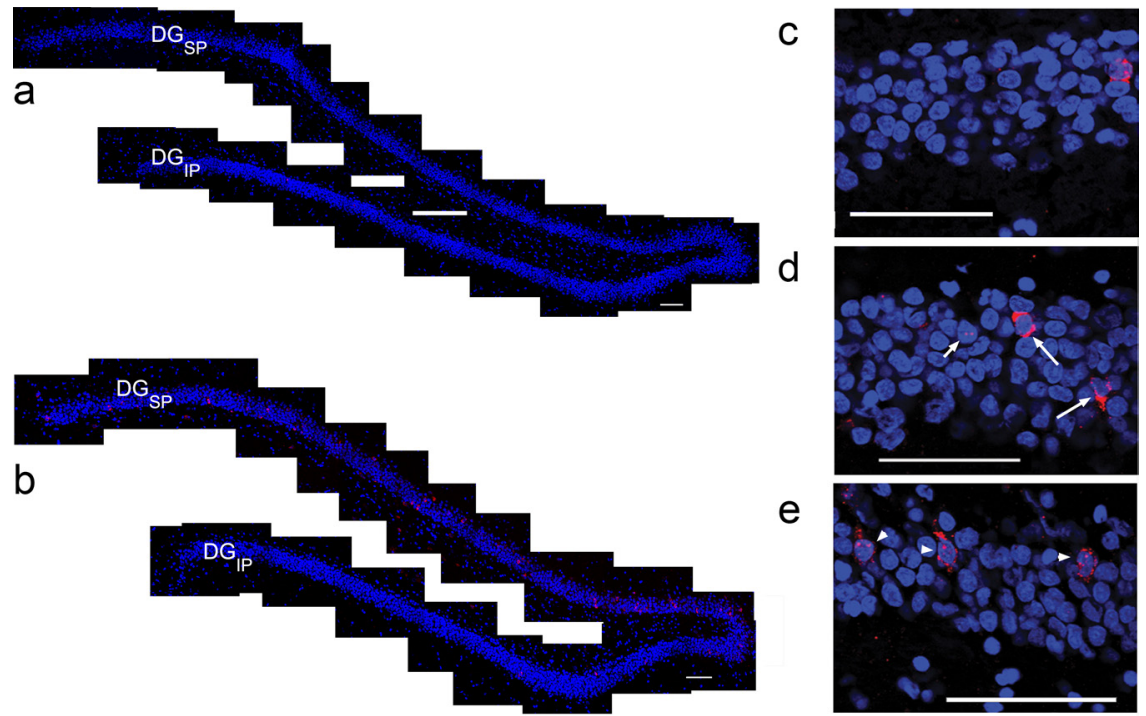

d

e

Figure 2. The pattern of DG zif268 expression is altered by task demands. $\boldsymbol{a}, \boldsymbol{b}$, Representative montages of confocal images of the DG demonstrate little zif268 expression in caged control animals $(\boldsymbol{a})$, whereas navigation of the plus maze $(\boldsymbol{b})$ induces a robust increase in zif268 localized in the suprapyramidal $\left(D_{S \mathrm{SP}}\right)$ but not the infrapyramidal $\left(D_{\mid P}\right)$ blade. $c-e$, Higher-magnification suprapyramidal DG micrographs illustrate the differences in zif268 transcription patterns in the three behavioral conditions. Compared to caged controls $(\boldsymbol{c})$, the granule cells of animals that navigated the same maze using different strategies ( $\boldsymbol{d}$ ) expressed zif268 solely within the nucleus (short arrow) or cytoplasm (long arrows), and granule cells in animals that used the same strategy (e) expressed zif268 predominantly within both compartments (arrowheads). Granule cell nuclei are counterstained with DAPI (blue) and zif268 is labeled with Cy3 (red). Scale bars: $\boldsymbol{a}, \boldsymbol{b}, 200 \mu \mathrm{m} ; \boldsymbol{c}, \boldsymbol{d}, \boldsymbol{e}, 60 \mu \mathrm{m}$.

a
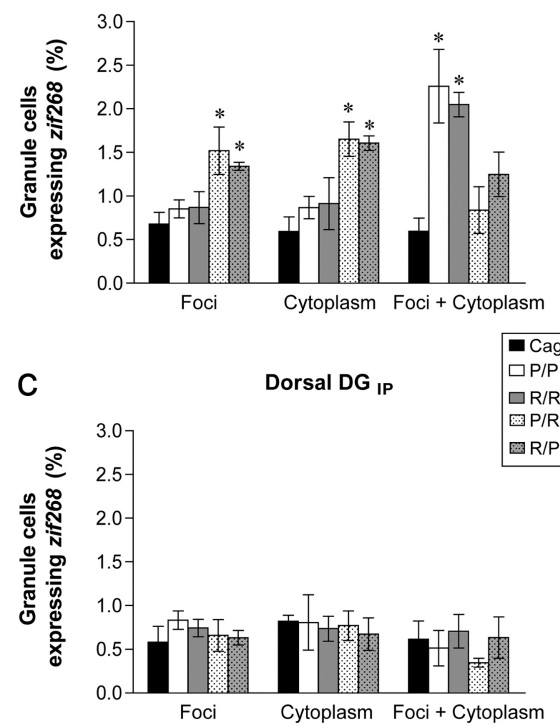

b

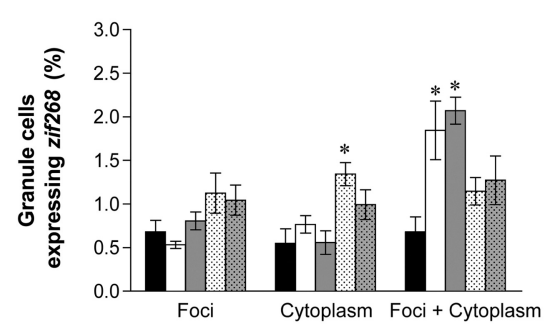

d

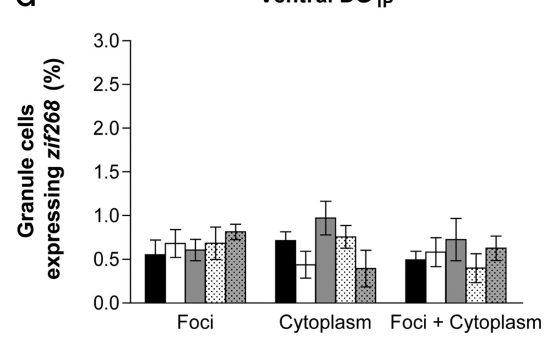

Figure 3. Granule cells expressing zif268 across the DG. Within the suprapyramidal blade (DG $G_{\mathrm{SP}}$ ) of both the dorsal $(\boldsymbol{a})$ and ventral ( $\boldsymbol{b}) D G$, rats that navigated the plus maze using different strategies $(P / R$ and $R / P)$ consistently had more granule cells expressing zif268 within either the nucleus (Foci) or the cytoplasm. These results indicate that unique populations of granule cells were recruited to express zif268 during each navigation session. In contrast, granule cells of animals that navigated the maze using the same strategy twice (P/P and R/R) expressed zif268 in both compartments (Foci + Cytoplasm), showing that the same cells were active during both navigation sessions. In the infrapyramidal blade $\left(\mathrm{DG}_{\mid \mathrm{P}}\right)$, no differences were observed in either the dorsal (c) or ventral (d) DG (mean \pm SEM; ${ }^{*} p<0.05$, relative to caged).

Using MetaMorph software (Molecular Devices), granule cells through the median $20 \%$ of each stack were classified as follows: zif268-negative, zif268-positive within the nucleus, zif268-positive within the cytoplasm, and zif268-positive in both the nucleus and cytoplasm. An average of 1878 granule cells (range, 990-3883) were counted per animal. The localization of zif268 (Fig. 1d) provides a histological marker of neurons engaging
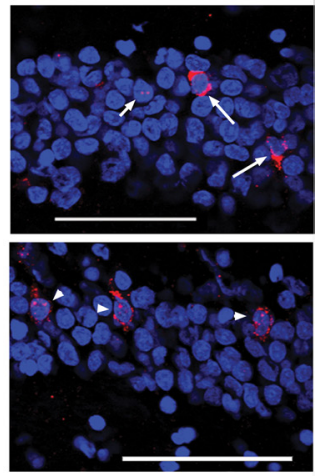

in transcription $30 \mathrm{~min}$ (producing cytoplasmic zif268) and 5 min (producing intranuclear zif268) before decapitation.

\section{Statistical analysis}

The pattern of zif268 expression was compared across groups in the dorsal and ventral suprapyramidal and infrapyramidal blades using one-way ANOVA, followed by Tukey's HSD post hoc tests. In addition, the proportion of granule cells repeatedly transcribing zif268 in animals that performed either the same strategy tasks $(\mathrm{R} / \mathrm{R}$ and $\mathrm{P} / \mathrm{P})$ or different tasks $(\mathrm{P} / \mathrm{R}$ and $\mathrm{R} / \mathrm{P}$ ) and was compared using $t$ tests.

\section{Results}

\section{Granule cells within the} suprapyramidal blade respond to changes in task demands

In the suprapyramidal blade of the dorsal DG, all four groups that navigated the plus maze expressed zif268 in significantly more granule cells than caged controls. However, the pattern of zif268 expression depended upon the behavioral group (Fig. $3 a)$. These groups differed significantly in the number of granule cells expressing zif268 within the nucleus $\left(F_{(4,17)}=5.31\right.$; $p=0.006)$. Post hoc tests demonstrated that animals in both the $\mathrm{P} / \mathrm{R}$ and $\mathrm{R} / \mathrm{P}$ groups had more granule cells expressing intranuclear zif268 $(p<0.05)$ than caged controls. In contrast, neither $\mathrm{P} / \mathrm{P}$ nor $\mathrm{R} / \mathrm{R}$ groups differed from controls $(p>0.10)$. Similarly, the experimental groups differed significantly in the number of cells expressing cytoplasmic zif268 $\left(F_{(4,17)}=\right.$ 7.23; $p=0.001)$ and post hoc tests showed that only $\mathrm{P} / \mathrm{R}(p=0.003)$ and $\mathrm{R} / \mathrm{P}(p=$ $0.002)$ groups significantly differed from controls (P/P and R/R, $p>0.10)$.

In addition, although the number of granule cells expressing zif268 in both the nucleus and the cytoplasm significantly differed between groups $\left(F_{(4,17)}=7.88\right.$; $p=0.001)$, post hoc tests revealed a unique pattern of results. In this case, $\mathrm{P} / \mathrm{P}(p=$ 0.002 ) and $\mathrm{R} / \mathrm{R}(p=0.007)$ groups expressed zif268 in significantly more cells than control animals, whereas $\mathrm{P} / \mathrm{R}$ and $\mathrm{R} / \mathrm{P}$ groups did not $(p>0.10)$.

The suprapyramidal blade of the ventral DG showed a similar pattern of results (Fig. 3b). A significant effect of group was apparent on the number of cells expressing cytoplasmic zif268 $\left(F_{(4,17)}=5.76 ; p=\right.$ $0.004)$ and a similar, although nonsignificant $\left(F_{(4,17)}=2.55 ; p=0.07\right)$, trend was seen in the number of cells expressing intranuclear zif268. Although animals engaging in two different strategies $(\mathrm{P} / \mathrm{R}$ and $\mathrm{R} / \mathrm{P})$ had more granule cells expressing zif268 in either the nucleus or cytoplasm relative to controls, these differences were not significant in post hoc tests (all $p>0.10$ ), with the exception of cytoplasmic zif268 expression in P/R animals 


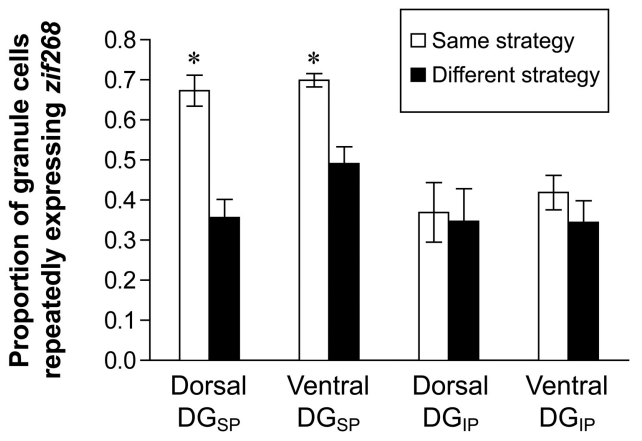

Figure 4. Overlap in repeated zif268 expression in the DG. When the same navigational strategy is used twice, $\sim 70 \%$ of granule cells in the suprapyramidal blade $\left(D G_{S p}\right)$ that expressed zif268 during the first session also expressed it in the second session. This overlap is significantly greater than is seen in animals that navigated the plus maze using two different navigational strategies, showing that changing task demands recruited a distinct population of granule cells. In the infrapyramidal blade $\left(D G_{\mid p}\right)$, no differential recruitment was apparent (mean \pm SEM; ${ }^{*} p<0.05$, same strategy vs different strategy within the same region).

( $p=0.01$ ). In addition, the number of cells expressing zif268 in both cellular compartments significantly differed between groups $\left(F_{(4,17)}=7.15, p=0.001\right)$ and post hoc tests revealed that only $\mathrm{P} / \mathrm{P}(p=0.006)$ and $\mathrm{R} / \mathrm{R}(p=0.001)$ were significantly different from caged controls $(\mathrm{P} / \mathrm{R}$ and $\mathrm{P} / \mathrm{P}, p>0.10)$.

These data show that the pattern of zif268 expression across the suprapyramidal blade can change dramatically as a result of the behavioral strategy. In animals that navigated the plus maze twice using the same strategy, the probability that a single granule cell would transcribe zif268 repeatedly during both the first and second session was $\sim 70 \%$. If, however, the rat engaged in two different navigational strategies, this probability decreased significantly in both the dorsal $\left(36 \% ; t_{(15)}=5.33 ; p<0.001\right)$ and ventral $\left(49 \%\right.$; $t_{(15)}$ $=4.40 ; p<0.001$ ) suprapyramidal blade (Fig. 4 ).

\section{Granule cells within the infrapyramidal blade show little response}

In the infrapyramidal blade of both the dorsal (Fig. 3c) and ventral (Fig. 3d) DG, there was no significant effect on zif268 expression within the nucleus, within the cytoplasm, or within both compartments (all cases: $F_{(4,17)}<2.00, p>0.10$ ). Similarly, the probability that individual granule cells repeatedly transcribed zif268 during both sessions was not significantly different between groups in either the dorsal $\left(t_{(15)}=1.06 ; p>0.10\right)$ or ventral $\left(t_{(15)}=1.68 ; p>0.10\right)$ infrapyramidal blade. These data indicate that only constitutive transcription was present in this region.

\section{Discussion}

Different task demands or behavioral strategies, independent of spatial location and trajectory, induce distinct patterns of zif268 expression within the suprapyramidal blade of both the DG. That is, when rats performed the same strategy twice (P/P and $R / R)$, zif268 was repeatedly expressed in the same population of granule cells. However, navigation by two different strategies (P/R and $\mathrm{R} / \mathrm{P}$ ) in the same trajectory within the same maze induced zif268 in distinct ensembles of granule cells. Notably, the tasks were designed so that sensory stimuli, trajectory direction, velocity, acceleration, motivational states, and the space occupied by the rats were identical for the two navigation sessions; the only apparent difference between the two tasks was the strategy that the rats adapted to successfully receive reward during each session. The observed changes in zif268 expression show that the DG is involved in the formation of distinct representations of different mnemonic aspects of an event, in addition to spatial context.

Because spatial firing patterns in pyramidal cells change with mnemonic demands, it has been suggested that the hippocampus mediates multiple representations of the same location so that different tasks within that space may be distinguished (Smith and Mizumori, 2006). The present study suggests that the DG may be critical for creating such differential representations.

Although changing task demands clearly alter granule cell recruitment, it remains possible that this differential encoding originates upstream of the DG. Previous data strongly suggest that pattern separation does not originate in the medial entorhinal cortex (Fyhn et al., 2007; Leutgeb et al., 2007). The possible role of other DG inputs (e.g., lateral entorhinal cortex, the medial septum, the median raphe, ventral tegmental area, supramammillary projections) in DG pattern separation has yet to be addressed.

Although very little is known directly about the firing patterns that are sufficient to induce zif268 in granule cells during behavior, patterns of stimulation sufficient to induce long-term plasticity reliably induce zif268, and zif268 expression is necessary for plasticity to become long-lasting (Jones et al., 2001). Moreover, recent data show that the firing inherent to an animal running a single lap through a track, and thus likely a single pass through a place field (a very small number of spikes), is sufficient to induce robust IEG expression (Miyashita et al., 2009).

Consequently, it is probable that all cells engaged in spatially tuned firing in the current tasks expressed zif268. Other factors that could contribute to pattern separation such as changes in firing rates or locations of firing fields would be expected not to alter the expression of zif268. Thus, zif268 only provides an index of changes in granule cell recruitment and as such a conservative/ minimal estimate of pattern separation. Importantly, the patterns of zif268 expression observed here is remarkably similar to the pattern of gene expression in the granule cells following exploration of two distinct spatial locations (Chawla et al., 2005; Marrone et al., 2010), a situation where granule cells have nearly zero temporal correlation in the firing of cell pairs (Leutgeb et al., 2007). Together, the current data show that changes in task demands within a single spatial location can reliably alter granule cell recruitment.

Not unexpectedly, the changes observed in the expression of zif268 were only manifested in the suprapyramidal blade. The two sessions in the plus maze induced only a modest number of granule cells to express zif268 both in the dorsal and ventral infrapyramidal blade, an amount that was comparable to caged control animals. These results are consistent with several previous reports describing no transcriptional response in the infrapyramidal blade immediately following spatial behavior (Chawla et al., 2005; Ramirez-Amaya et al., 2006; Vazdarjanova et al., 2006). A functional gradient has also been proposed from spatial processing at the septal pole to either nonspatial (Moser and Moser, 1998) or more emotional (Segal et al., 2010) processing at the temporal pole. Our data indicate a similar degree of responsiveness to task demands in both the septal and temporal DG. This differs from the selective remapping observed in dorsal but not ventral CA1 (Markus et al., 2010). Thus, the responsiveness of pyramidal cells along the longitudinal axis is likely mediated by direct entorhinal and/or subcortical inputs.

In sum, the present study show that tasks with different mnemonic demands recruit distinct populations of granule cells. The encoding and retrieval of each event in distinct populations of granule cells may prevent interference between the tasks required in the same location and permit the selection of the appropriate 
responses. This differential encoding is consistent with the role the hippocampus plays in episodic memory (Eichenbaum et al., 1999) and suggests that the DG makes a unique contribution to the orthogonalization of episodic memories, as it disambiguates events that differ in cognitive demands, independent of other aspects of behavior.

\section{References}

Alme CB, Buzzetti RA, Marrone DF, Leutgeb JK, Chawla MK, Schaner MJ, Bohanick JD, Khoboko T, Leutgeb S, Moser EI, Moser MB, McNaughton BL, Barnes CA (2010) Hippocampal granule cells opt for early retirement. Hippocampus 20:1109-1112.

Amaral DG, Ishizuka N, Claiborne B (1990) Neurons, numbers and the hippocampal network. Prog Brain Res 83:1-11.

Bostock E, Muller RU, Kubie JL (1991) Experience-dependent modifications of hippocampal place cell firing. Hippocampus 1:193-205.

Chawla MK, Guzowski JF, Ramirez-Amaya V, Lipa P, Hoffman KL, Marriott LK, Worley PF, McNaughton BL, Barnes CA (2005) Sparse, environmentally selective expression of Arc RNA in the upper blade of the rodent fascia dentata by brief spatial experience. Hippocampus 15:579-586.

Eichenbaum H, Dudchenko P, Wood E, Shapiro M, Tanila H (1999) The hippocampus, memory, and place cells: is it spatial memory or a memory space? Neuron 23:209-226.

Fyhn M, Hafting T, Treves A, Moser MB, Moser EI (2007) Hippocampal remapping and grid realignment in entorhinal cortex. Nature 446:190-194.

Gilbert PE, Kesner RP, Lee I (2001) Dissociating hippocampal subregions: double dissociation between dentate gyrus and CA1. Hippocampus 11:626-636.

Guzowski JF, McNaughton BL, Barnes CA, Worley PF (1999) Environmentspecific expression of the immediate-early gene Arc in hippocampal neuronal ensembles. Nat Neurosci 2:1120-1124.

Jones MW, Errington ML, French PJ, Fine A, Bliss TV, Garel S, Charnay P, Bozon B, Laroche S, Davis S (2001) A requirement for the immediate early gene Zif268 in the expression of late LTP and long-term memories. Nat Neurosci 4:289-296.

Jung MW, McNaughton BL (1993) Spatial selectivity of unit activity in the hippocampal granular layer. Hippocampus 3:165-182.

Kennedy PJ, Shapiro ML (2009) Motivational states activate distinct hippocampal representations to guide goal-directed behaviors. Proc Natl Acad Sci U S A 106:10805-10810.

Leutgeb JK, Leutgeb S, Moser MB, Moser EI (2007) Pattern separation in the dentate gyrus and CA3 of the hippocampus. Science 315:961-966.

Leutgeb S, Leutgeb JK, Barnes CA, Moser EI, McNaughton BL, Moser MB (2005) Independent codes for spatial and episodic memory in hippocampal neuronal ensembles. Science 309:619-623.

Markus EJ, Qin YL, Leonard B, Skaggs WE, McNaughton BL, Barnes CA (1995) Interactions between location and task affect the spatial and directional firing of hippocampal neurons. J Neurosci 15:7079-7094.

Markus EJ, Schmidt B, Satvat, E, LaMontage T, Som C, Marrone DF (2010) Regional hippocampal expression of IEGs during a spatial and nonspatial navigation task with an identical trajectory. Soc Neurosci Abstr 36:709.8.
Marr D (1971) Simple memory: a theory for archicortex. Philos Trans R Soc Lond B Biol Sci 262:23-81.

Marrone DF, Adams AA, Satvat E (2010) Increased pattern separation in the aged fascia dentata. Neurobiol Aging. Advance online publications. Retrieved January 5, 2011. doi:10.1016/j.neurobiolaging.2010.03.021.

McNaughton BL, Morris RG (1987) Hippocampal synaptic enhancement and information storage within a distributed memory system. Trends Neurosci 10:408-415.

Miyashita T, Kubik S, Haghighi N, Steward O, Guzowski JF (2009) Rapid activation of plasticity-associated gene transcription in hippocampal neurons provides a mechanism for encoding of one-trial experience. J Neurosci 29:898-906.

Moser MB, Moser EI (1998) Functional differentiation in the hippocampus. Hippocampus 8:608-619.

O'Keefe J, Nadel L (1978) The hippocampus as a cognitive map. Oxford: Oxford UP.

Paxinos G, Watson C (2004) The rat brain in stereotaxic coordinates, fourth edition. New York: Elsevier.

Quirk GJ, Muller RU, Kubie JL (1990) The firing of hippocampal place cells in the dark depends on the rat's recent experience. J Neurosci 10:2008-2017.

Ramirez-Amaya V, Marrone DF, Gage FH, Worley PF, Barnes CA (2006) Integration of new neurons into functional neural networks. J Neurosci 26:12237-12241.

Rolls ET, Kesner RP (2006) A computational theory of hippocampal function, and empirical tests of the theory. Prog Neurobiol 79:1-48.

Schmidt B, Jacobson TK, Markus E (2009) Hippocampal and striatal dependent navigation: sex differences are limited to acquisition. Horm Behav 56:199-205.

Segal M, Richter-Levin G, Maggio N (2010) Stress-induced dynamic routing of hippocampal connectivity: a hypothesis. Hippocampus 20:1332-1338.

Skaggs WE, McNaughton BL (1998) Spatial firing properties of hippocampal CA1 populations in an environment containing two visually identical regions. J Neurosci 18:8455-8466.

Smith DM, Mizumori SJ (2006) Hippocampal place cells, context, and episodic memory. Hippocampus 16:716-729.

Treves A, Rolls ET (1992) Computational constraints suggest the need for two distinct input systems to the hippocampal CA3 network. Hippocampus 2:189-199.

Vazdarjanova A, Ramirez-Amaya V, Insel N, Plummer TK, Rosi S, Chowdhury S, Mikhael D, Worley PF, Guzowski JF, Barnes CA (2006) Spatial exploration induces ARC, a plasticity-related immediate-early gene, only in calcium/calmodulin-dependent protein kinase II-positive principal excitatory and inhibitory neurons of the rat forebrain. J Comp Neurol 498:317-329.

Wills TJ, Lever C, Cacucci F, Burgess N, O’Keefe J (2005) Attractor dynamics in the hippocampal representation of the local environment. Science 308:873-876.

Wood ER, Dudchenko PA, Robitsek RJ, Eichenbaum H (2000) Hippocampal neurons encode information about different types of memory episodes occurring in the same location. Neuron 27:623-633. 